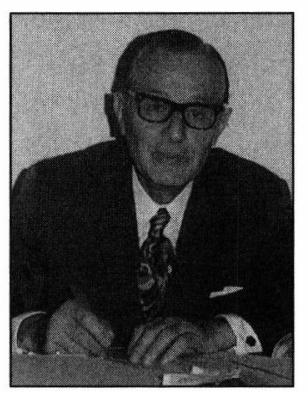

\title{
ÉDITORIAL
}

\section{TRENTIÈME ANNIVERSAIRE DE L'ASSOCIATION}

Monsieur le Professeur André Morette, titulaire de la Chaire d'Hydrologie, le 13 février 1969, dans la salle des Actes de la faculté de Pharmacie de Paris, fit la première "réunion-exposition» destinée aux spécialistes Universitaires et extraUniversitaires, réunion, intitulée: préservation de l'intégrité de l'eau dans la nature.

Comment l'idée de notre Association a-t-elle pris corps il y a 30 ans. Où et quand a germé cette idée de notre président fondateur?

C'est en vérité le Week-End de la pentecôte juste après l'épopée du bouillonnement des idées de mai 1968. En effet c'était le premier dimanche où l'essence revenait avec abondance dans les pompes et qu'ainsi de nombreux parisiens pouvaient prendre l'air des pré-vacances estivales et se précipitaient dans les forêts des environs de Paris comme chaque année d'ailleurs. Il en fut ainsi de Monsieur et Madame Morette et de Monsieur et Madame Trannoy qui se rencontrèrent fortuitement à Milly-La-Forêt autour du célèbre lavoir. Monsieur Robert Trannoy venait de terminer une thèse dans le laboratoire de Monsieur Morette et travaillait, comme cadre Pharmacien, au sein de la société industrielle pharmaceutique ClinByla.

Monsieur Morette, toujours chaleureux avec ses anciens élèves s'entretint de ses idées souvent novatrices, sur le monde en général, sur l'eau et la santé en particulier, domaine dans lequel il travaillait avec passion, à la Faculté de Pharmacie de Paris qui lui était d'autre part si chère. Ainsi dit-il à Monsieur Trannoy qu'il 
fallait absolument rebondir sur cette effervescence de la Faculté en 1968 et créer, pourquoi pas, une Association avec trois partenaires: la Faculté, l'Industrie de l'Eau et l'Industrie Pharmaceutique, c'est-à-dire je le cite: «des spécialistes intéressés par l'eau et la protection de sa pureté et de sa disponibilité à l'orée de ce XXIe siècle, socio-économico industriel, à la fois, serviable et dur aux hommes ». Monsieur Morette était un chimiste de formation et il aimait comparer de nombreuses situations humaines aux réactions chimiques, aussi disait-il sur cette naissance de l'Association, qu'elle était le résultat d'échanges incessants de matière dans l'univers, fait de l'infinité des contacts et des chocs qui provoquent la naissance de nouveaux mondes, qu'elle pouvait provenir d'un processus de nucléation à partir de multiples idées, ou bien d'un transfert incessant des germes dans un liquide qui provoque l'apparition des cybomas puis la germination d'un cristal.

Monsieur Trannoy apporta aussitôt son soutient enthousiaste en tant qu'industriel chez Clyn-Bila et se proposa comme trésorier. L'idée était donc née et Monsieur Morette prit son bâton de pèlerin avec ténacité, une de ses qualité, entreprit de recruter des personnalités disponibles et compétentes afin de faire fonctionner cette structure. Monsieur Jehan Gauduchon, directeur à l'époque de l'usine Clin Byla de Gentilly, directeur de la qualité Clin Midy, membre de l'Académie de Phamacie avait déjà beaucoup ouvré pour des Associations telles que celle des anciens élèves de l'Institut Pasteur et fût ainsi chargé de rédiger des statuts et un règlement intérieur. Ce qu'il fit volontiers. Ces textes sont toujours en vigueur tels quels.

Monsieur Roger Cabridenc de l'IRCHA (Institut de Recherche de Chimie Appliquée) fut chargé du secrétariat. Monsieur Syboni de la société OTV (Omnium des Techniques de valorisation) représenta les industriels de l'eau.

Monsieur Jean Bontoux de Montpellier apporta sa compétence dans le domaine des eaux usées, Monsieur Arnoux dans celui de la Mer puisqu'il enseignait à la Faculté de Pharmacie de Marseille, Monsieur Jean Canellas de Bordeaux pour son immense savoir en ce qui concerne les eaux thermales.

Monsieur Guy Laporte, pharmacien à Thiais, participa également aux premiers pas de l'Association, il avait étudié l'influence de l'eau dans la conservation des fresques de Lascaut, puis décida avec d'autres spécialistes leur fermeture au public pour les sauvegarder avec reconstruction à coté d'une grotte identique.

Ainsi fonctionna sur le papier notre Association avec ces quelques éminentes personnalités compétentes, dévoués et prêtes à se lancer dans ce travail ardu et ingrat du point de vue matériel quant à sa réalisation avec les premiers moyens de l'époque.

Puis les séances de l'Association vont commencer d'être régulièrement organisées à Paris 2 ou 3 fois par an.

La première séance scientifique du 11 mai 1970 avait pour programme:

- Les détergents, problèmes posés, pollution, aspects pratiques et scientifiques par Roger Cabridenc puis, 
- Recherche des entérovirus par Messieurs Jean-Marie Foliguet et Louis Schwartzbrood de Nancy et enfin,

- Les échangeurs d'ions et la pollution des eaux. Messieurs de Saint Léon Langlès, et Berrely.

La deuxième séance de l'année, le 19 octobre 1970 avait entre autre pour programme :

- Recherche sur la cytotoxicité par le Docteur L. Coin et Mme Guinot

- Rejet en mer des détergents par Mr. Arnoux

- Interférences des acides gras dans le dosage des hydrocarbures aliphatiques par spectrométrie infrarouge. M. Paul Chambon actuel Président de notre Association et Mme Chambon

Ensuite ces journées annuelles furent consacrées à un seul thème comme celui de la radioactivité en 1972 avec Messieurs J. Rodier, Bizollon, Saumande.

Très vite, le besoin d'un journal périodique se fit sentir, à la fois pour établir un lien supplémentaire entre les adhérents et promouvoir un support de recherches sur l'eau à travers la France, d'autant que à l'époque les journaux existant sous cette forme étaient peu nombreux dans le domaine de l'Hydrologie Analytique.

Avec les moyens de la Faculté, c'est un journal rudimentaire dans sa forme qui fut fabriqué, d'abord au laboratoire où tout le personnel participa à son élaboration (Melle Desvallons sa secrétaire, Mmes Monique Rambaud et Jeanine Rouault, techniciennes, enfin Melle Nicole Strüpler et moi-même, enseignants à paris V). Ensuite, avec le temps un imprimeur dans le 14e, continua l'impression du Journal en l'améliorant. Les financements furent obtenus à la fois des cotisants, des industriels de l'eau ou du Conseil de l'Ordre et de la profession pharmaceutique. En voulant attendre tous les manuscrits relatifs à un même thème, le retard dans la parution était chronique.

Vous avez ici, dans cette salle des Actes, tous les numéros que j'ai pu rassembler, de l'origine à nos jours sans exception, ce qui représente une soixantaine de numéros.

Vous pourrez constater l'évolution considérable de la revue en espérant que cette évolution ne s'arrêtera pas là et pour reprendre les propres paroles de monsieur Morette il y a 30 ans, je cite «espérons que le destin de notre association n'aura rien d'éphémère et qu'au contraire celui-ci sera de faire œuvre utile et précieuse par le truchement de la Pharmacie».

Je termine en remerciant Monsieur Paul Chambon l'actuel Président qui permet à l'Association de remplir son rôle édicté en 1969, tous les présents d'aujourd'hui dans cette salle des Actes, ainsi que tous ceux qui ont œuvré dans l'ombre parfois, mais avec leur concours pratique et précieux, et enfin tous les cotisants de notre Association petits et grands dont certains sont restés d'une fidélité exemplaire pendant 30 ans. 
Je remercie également madame Morette-Maillant ici présente, la fille de monsieur Morette, qui bien qu'ayant toujours travaillé dans le domaine de la sauvegarde du patrimoine au Ministère de la culture s'est fort intéressée au devenir de l'association de son père et $m$ 'a fourni des photos et des renseignements très précieux.

De même Monsieur Robert Trannoy, parmi les tous premiers acteurs de notre Association, qui n'a pas manqué beaucoup de séances et nous honore encore aujourd'hui même, de sa participation

Comme Monsieur Morette qui était revenu tout émerveillé d'un voyage aux Etats Unis en 1951 pour la Pharmacie, je souhaite que l'Association puisse se rendre prochainement non pas si loin mais dans un pays européen où elle pourrait accueillir en son seing d'éminents spécialistes de l'eau et de la santé, de la Communauté, et s'enrichir scientifiquement.

Paris, le 5 mai 1999

Gilles HussoN

Trésorier

Rédacteur-en-Chef du Journal Européen d'Hydrologie

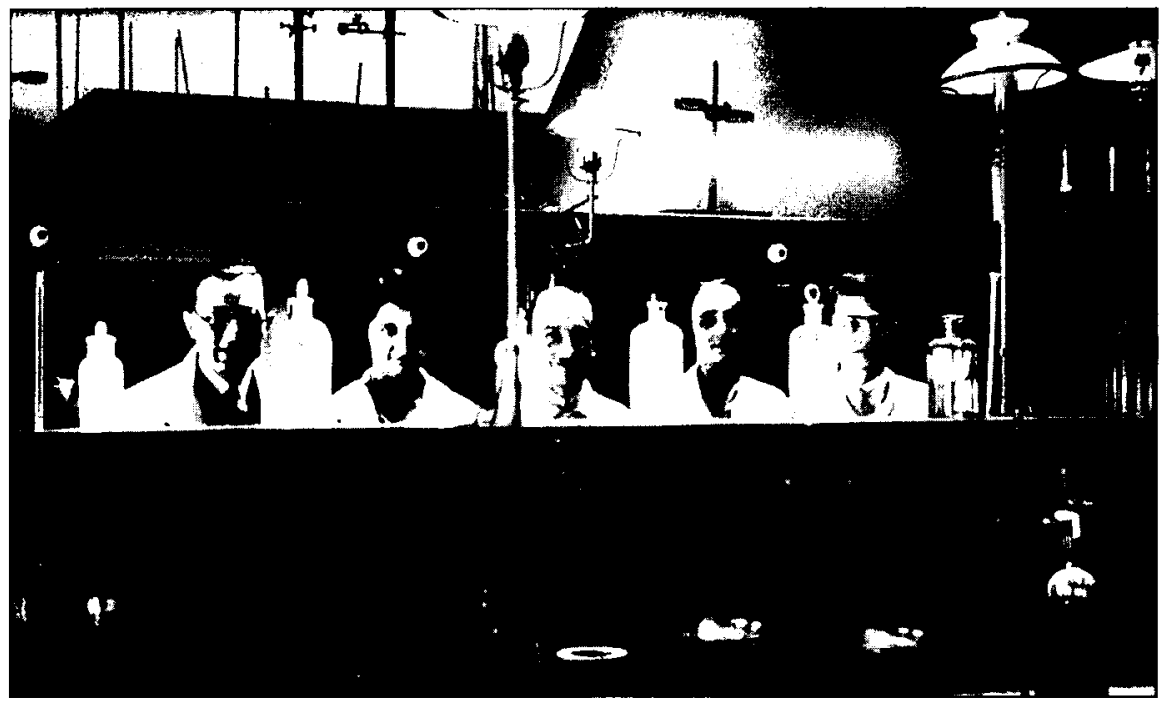

Réunion des Professeurs.

Faculté de Pharmacie (1964), M. Morette (1er à gauche). 